\title{
Complete Genome Sequence of the First Chrysovirus From the Phytopathogenic Fungus Alternaria Solani on Potato in China
}

Chenghui Hu

China Agricultural University

Siwei Li

China Agricultural University

Chunyan Wu

China Agricultural University

Yiran Mi

China Agricultural University

Qingnian Cai

China Agricultural University

\section{Tao Zhou}

China Agricultural University

\section{Can Zhao}

China Agricultural University

Xuehong Wu ( $\nabla$ wuxuehong@cau.edu.cn)

China Agricultural University https://orcid.org/0000-0003-2669-2300

\section{Research Article}

Keywords: Chrysoviridae, Alphachrysovirus, Phylogenetic analysis, Potato, Alternaria solani, Untranslated region (UTR)

Posted Date: July 19th, 2021

DOl: https://doi.org/10.21203/rs.3.rs-721752/v1

License: (c) (i) This work is licensed under a Creative Commons Attribution 4.0 International License.

Read Full License

Version of Record: A version of this preprint was published at Archives of Virology on October 8th, 2021. See the published version at https://doi.org/10.1007/s00705-021-05263-z. 


\section{Abstract}

The full genome of a double-stranded RNA (dsRNA) mycovirus, which was isolated from Alternaria solani strain DT-10 causing potato foliar disease and designated as Alternaria solani chrysovirus 1 (AsCV1), consisted of four dsRNA segments (dsRNA 1-4) with the length of $3600 \mathrm{bp}, 3128 \mathrm{bp}, 2996 \mathrm{bp}$, and 2714 bp, respectively. RNA-dependent RNA polymerase (RdRp, 1084 amino acids (aa)), putative capsid protein (905 aa), Alphachryso-P3 (835 aa), and Alphachryso-P4 (729 aa) were encoded by dsRNA1, dsRNA2, dsRNA3, and dsRNA4, respectively, which had the highest identities of $41.77 \%-72.38 \%$ to the counterparts of Helminthosporium victoriae virus $145 S$ (HvV145S) in the genus Alphachrysovirus of the family Chrysoviridae. Moreover, the 5 '-untranslated regions (UTRs) with several unique inserts (3-37 bp) and deletions (5-64 bp) of AsCV1 dsRNA 1-4 shared 51.65\%-68.01\% identities to those of HvV145S. Phylogenetic analysis of RdRp suggested that AsCV1 clustered the most closely with HvV145S. Based on the characteristics of distinct host, low identities of encoded proteins, special traits in 5'-UTRs of dsRNA $1-4$, and phylogenetic analysis of RdRp, AsCV1 was confirmed to be a new species in the genus Alphachrysovirus. To our best knowledge, this is the first alphachrysovirus identified from phytopathogenic $A$. solani.

\section{Introduction}

Mycoviruses identified in fungi and oomycetes, have genomic types of double-stranded RNA (dsRNA), single-stranded RNA (ssRNA), and DNA [1]. DsRNA mycoviruses belong to seven families including Birnaviridae, Chrysoviridae, Cystoviridae, Megabirnaviridae, Partitiviridae, Picobirnaviridae, and Quadriviridae, and a new established genus Botybirnavirus [2, 3]. The family Chrysoviridae contains two genera, namely Alphachrysovirus (17 species) and Betachrysovirus (8 species). Members of Chrysoviridae possess 3-7 dsRNA segments with each segment being separately encapsulated. Among these dsRNA segments, the dsRNA1 and dsRNA2 encodes RNA-dependent RNA polymerase (RdRp) and capsid protein (CP), respectively. Virions of chrysoviruses, whose hosts include fungi, plants, and insects, are isometric and non-enveloped, with the size of about $40 \mathrm{~nm}$ in diameter $[4,5]$.

In 1988, viral dsRNAs were detected in Alternaria alternata isolated from Japanese pear trees, which is the first reported mycovirus in Alternaria [6]. Later, many mycoviruses had been identified in A. alternata, such as Alternaria alternata virus-1 (AAV-1) [7], Alternaria alternata chrysovirus 1 (AaCV1) [8], Alternaria alternata partitivirus 1 (AtPV1) [9], Alternaria alternata botybirnavirus 1 (AaBRV1) [3], and Alternaria alternata hypovirus 1 (AaHV1) [10]. In addition, several mycoviruses including Alternaria tenuissima partitivirus 1 (AttPV1) [11], Alternaria brassicicola endornavirus 1 (AbEV1) [12], Alternaria longipes dsRNA virus 1 (AIRV1) [13], Alternaria arborescens victorivirus 1 (AaVV1) [14], and Alternaria dianthicola dsRNA virus 1 (AdRV1) [15] had been reported in five other species (A. tenuissima, A. brassicicola, A. longipes, $A$. arborescens, and $A$. dianthicola) of Alternaria. Recently, an ssRNA mycovirus named Alternaria solani fusarivirus 1 (AsFV1) had been isolated from $A$. solani [16]. 
In our previous study, A. solani had been documented to cause potato early blight in China $[17,18]$. In this study, A. solani strain DT-10, which was recovered from diseased leaves of potato with the symptoms of early blight in Datong city, Shanxi province of China, was identified according to the methods described previously $[17,18]$. A new alphachrysovirus was found to be associated with the $A$. solani strain DT-10 and designated as Alternaria solani chrysovirus 1 (AsCV1).

\section{Provenance of the virus material}

Alternaria solani strain DT-10 was cultured on potato dextrose agar (PDA) plates overlaid with cellophane membranes for 7 days in the dark at $25^{\circ} \mathrm{C}$. Multiple dsRNAs were extracted from the mycelia by binding to the CF-11 cellulose powder in 15\% ethanol as described by Morris and Dodds [19]. After treatment with S1 nuclease and DNase I (TaKaRa, Dalian, China), the dsRNAs were analyzed by $1.0 \%$ agarose gel electrophoresis (Fig. 1A) and purified with a gel extraction kit (Aidlab Biotechnologies, Beijing, China). The partial complementary DNA (CDNA) sequences of the dsRNA segments were obtained and assembled as described previously [20]. Using specific primers designed based on the obtained sequences of cDNA (Supplementary Table 1), the terminal sequences were determined by RNA-ligase-mediated rapid amplification of cDNA ends (RLM-RACE) method as described previously [21, 22]. The full genome sequences of AsCV1 were obtained through assembling all the partial CDNA and terminal sequences with DNAMAN 7.0 (Lynnon Biosoft, USA), and deposited into the GenBank database with the accession numbers of MW656210, MW656211, MW656212, and MW656213 for dsRNA1, dsRNA2, dsRNA3, and dsRNA4, respectively.

\section{Sequence properties}

The full sequence of the four dsRNA fragments are 3,600 bp, 3,128 bp, 2,996 bp, and 2,714 bp, respectively. The open reading frame (ORF) of each dsRNA sequence was analyzed using ORFfinder online tool (https://www.ncbi.nlm.nih.gov/orffinder/). Each dsRNA contains a single ORF, which was named ORF1, ORF2, ORF3, and ORF4 for dsRNA1, dsRNA2, dsRNA3, and dsRNA4, respectively (Fig. 1B). BLASTp search for the four ORFs resulted in the highest similarity to the counterparts of Helminthosporium victoriae virus $145 S$ (HvV145S) in the genus Alphachrysovirus of the family Chrysoviridae. ORF1 encoded a protein of 1084 amino acids (aa) (124.56 kDa) with $72.38 \%$ sequence similarity to the RdRp of HvV145S-A9 (GenBank No. NC_005978). The predicted RdRp domain at 351803 aa contained eight conserved motifs (Supplementary Fig. 1A). The protein encoded by ORF2 contained 905 aa $(102.29 \mathrm{kDa})$, which showed $61.40 \%$ sequence identity to the putative CP of Bipolaris maydis chrysovirus 1 (BmCV1) (GenBank No. KY489955), an isolate of HvV145S [23]. BLASTp also presented that ORF3-encoded protein (835 aa, $92.84 \mathrm{kDa}$ ) and ORF4-encoded protein (729 aa, $82.31 \mathrm{kDa})$ had $69.84 \%$ identity to Alphachryso-P3 of HvV145S-A9 and $41.77 \%$ identity to Alphachryso-P4 of BmCV1, respectively.

The 5'-untranslated regions (UTRs) and 3'-UTRs of AsCV1 were 242 bp and 103 bp long in dsRNA1, 312 bp and 98 bp long in dsRNA2, 317 bp and 171 bp long in dsRNA3, and 315 bp and 209 bp long in dsRNA4, respectively (Supplementary Table 2). Multiple alignments of the 5'-UTR and 3'-UTR sequences 
of the four dsRNAs were performed using DNAMAN 7.0 (Lynnon Biosoft). The 5'-UTRs presented strong sequence similarities in their internal parts, sharing strictly conserved terminal sequence of CGATAAAAACAAAAA. Meanwhile, the 3'-UTRs among the four dsRNA segments shared conserved GCTTTAAGTGT termini (Supplementary Fig. 1B). The 5'-UTRs and 3'-UTRs of AsCV1 dsRNA1-4 had $51.65 \%-68.01 \%$ and $30.04 \%-70.34 \%$ identities to those of HvV145S-A9 and BmCV1. Intriguingly, several unique inserts (3-37 bp) and deletions (5-64 bp) were found in 5'-UTRs of AsCV1, which were different from those of HvV145S-A9 and BmCV1 (Fig. 2).

The RdRp sequences of AsCV1 and 24 members of the family Chrysoviridae were analyzed by ClustalX 2.0 and MEGA 6.0 [22]. The phylogenetic tree constructed by the neighbor-joining algorithm with 1000 replicates based on the RdRp sequences showed that AsCV1 formed a well-supported taxonomic cluster with the members of the genus Alphachrysovirus and was closely related to HvV145S-A9 and BmCV1 (Fig. 1C).

Virion particles were extracted from the mycelium of $A$. solani strain DT-10 and stained according to the methods previously described [24, 3]. Viewed under transmission electron microscope (TEM) (JEM-1230, JEOL, Tokyo, Japan), the particles of AsCV1 were isometric with a diameter of approximately $40 \mathrm{~nm}$ (Supplementary Fig. 1C).

According to ICTV, the host of isolation, nucleotide and deduced amino acid sequence data, the length of genome segments, and 5'-UTRs are useful species demarcation criteria for the genus Alphachrysovirus [4]. Based on host fungus $A$. solani, low sequence identities of proteins encoded, unusual characteristics of 5'-UTRs, and phylogenetic relationships to known members of Alphachrysovirus, AsCV1 is a novel member in the genus Alphachrysovirus. This is the first report of the complete genome sequence of a new chrysovirus infecting $A$. solani.

\section{Declarations}

\section{Compliance with ethical standards}

Funding: This work was financially supported by National Key Research and Development Program of China (2018YFD0200803).

Conflict of interest: The authors declare that they have no conflict of interest.

Ethical approval: This study did not include experiments with human participants or animals performed by any of the authors.

Informed consent: Informed consent was obtained from all individual participants included in this study.

\section{References}


1. Xie JT, Jiang DH (2014) New insights into mycoviruses and exploration for the biological control of crop fungal diseases. Annu Rev Phytopathol 52:45-68

2. Ghabrial SA, Castón JR, Jiang D, Nibert ML, Suzuki N (2015) 50-plus years of fungal viruses. Virology 479:356-368

3. Ma G, Liang Z, Hua H, Zhou T, Wu X (2019) Complete genome sequence of a new botybirnavirus isolated from a phytopathogenic Alternaria alternata in China. Arch Virol 164:1225-1228

4. Kotta LI, Coutts R, Hillman BI, Jiang D, Kim DH, Moriyama H, Suzuki N, ICTV Report Consortium (2020) ICTV Virus Taxonomy Profile: Chrysoviridae. J Gen Virol 101:143-144

5. Luque D, Gómez-Blanco J, Garriga D, Brilot AF, González JM, Havens WM, Carrascosa JL, Trus BL, Verdaguer N, Ghabrial SA (2014) Cryo-EM near-atomic structure of a dsRNA fungal virus shows ancient structural motifs preserved in the dsRNA viral lineage. Proc Natl Acad Sci USA 111:76417646

6. Hayashi N, Tsuge T, Kobayashi H, Nishimura S (1988) The presence of double-stranded RNAs in Alternaria alternata Japanese pear pathotype and their participation in AK-toxin productivity. Ann Phytopathol Soc JPN 54:250-252

7. Aoki N, Moriyama H, Kodama M, Arie T, Teraoka T, Fukuhara T (2009) A novel mycovirus associated with four double-stranded RNAs affects host fungal growth in Alternaria alternata. Virus Res 140:179-187

8. Okada R, Ichinose S, Takeshita K, Urayama SI, Fukuhara T, Komatsu K, Arie T, Ishihara A, Egusa M, Kodama M, Moriyama H (2018) Molecular characterization of a novel mycovirus in Alternaria alternata manifesting two-sided effects: Down-regulation of host growth and up-regulation of host plant pathogenicity. Virology 519:23-32

9. Xavier A, Barros A, Godinho M, Zerbini F, Souza F, Bruckner F, Alfenas-Zerbini P (2018) A novel mycovirus associated to Alternaria alternata comprises a distinct lineage in Partitiviridae. Virus Res 244:21-26

10. Li H, Bian R, Liu Q, Yang L, Pang T, Salaipeth L, Andika I, Kondo H, Sun L (2019) Identification of a novel hypovirulence-inducing hypovirus from Alternaria alternata. Front Microbiol 10:1076

11. Liang Z, Wang X, Hua H, Cao W, Zou T, Zhao C, Wu X (2020) Full genome sequence of a new threesegment gammapartitivirus from the phytopathogenic fungus Alternaria tenuissima on cotton in China. Arch Virol 166:973-976

12. Shang H, Zhong J, Zhang R, Chen C, Gao B, Zhu H (2015) Genome sequence of a novel endornavirus from the phytopathogenic fungus Alternaria brassicicola. Arch Virol 160:1827-1830

13. Lin Y, Zhang H, Zhao C, Liu S, Guo L (2015) The complete genome sequence of a novel mycovirus from Alternaria longipes strain HN28. Arch Virol 160:577-580

14. Komatsu K, Katayama Y, Omatsu T, Mizutani T, Fukuhara T, Kodama M, Arie T, Teraoka T, Moriyama $\mathrm{H}$ (2016) Genome sequence of a novel victorivirus identified in the phytopathogenic fungus Alternaria arborescens. Arch Virol 161:1701-1704 
15. Hu Z, Guo J, Gao B, Zhong J (2020) A novel mycovirus isolated from the plant-pathogenic fungus Alternaria dianthicola. Arch Virol 165:2105-2109

16. Gong W, Liu H, Zhu X, Zhao S, Cheng J, Zhu H, Zhong J, Zhou Q (2021) Molecular characterization of a novel fusarivirus infecting the plant-pathogenic fungus Alternaria solani. Arch Virol. https://doi.org/10.1007/s00705-021-05105-y

17. Zheng HH, Zhao J, Wang TY, Wu XH (2015) Characterization of Alternaria species associated with potato foliar diseases in China. Plant Pathol 64:425-433

18. Zhao J, Ma GP, Liu YY, Wu XH (2018) Alternaria species infecting potato in southern China. Can J Plant Pathol 40:312-317

19. Morris TJ, Dodds JA (1979) Isolation and analysis of double-stranded RNA from virus-infected plant and fungal tissue. Phytopathology 69:854-858

20. Lyu RL, Zhang Y, Tang Q, Li YY, Cheng JS, Fu YP, Chen T, Jiang DH, Xie JT (2017) Two alphapartitiviruses co-infecting a single isolate of the plant pathogenic fungus Rhizoctonia solani. Arch Virol 163:515-520

21. Liu LJ, Wang QH, Cheng JS, Fu YP, Jiang DH, Xie JT (2015) Molecular characterization of a bipartite double-stranded RNA virus and its satellite-like RNA co-infecting the phytopathogenic fungus Sclerotinia sclerotiorum. Front Microbiol 6:406

22. Li S, Li Y, Hu C, Han C, Zou T, Zao C, Wu X (2020) Full genome sequence of a new mitovirus from the phytopathogenic fungus Rhizoctonia solani. Arch Virol 165:1719-1723

23. Gong M, Wang H, Jia D, Yan F, Zhang S (2019) Increased pathogenicity of the pathogen of corn southern leaf blight caused by a novel chrysovirus. Proc Annu Meet Chin Soc Plant Pathol (2019), Beijing, 242

24. Xiang J, Fu M, Hong N, Zhai LF, Xiao F, Wang GP (2017) Characterization of a novel botybirnavirus isolated from a phytopathogenic Alternaria Arch Virol 162:3907-3911

\section{Figures}


A

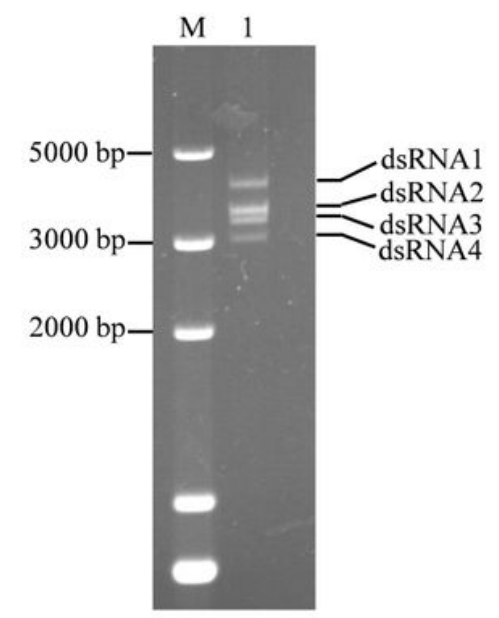

B

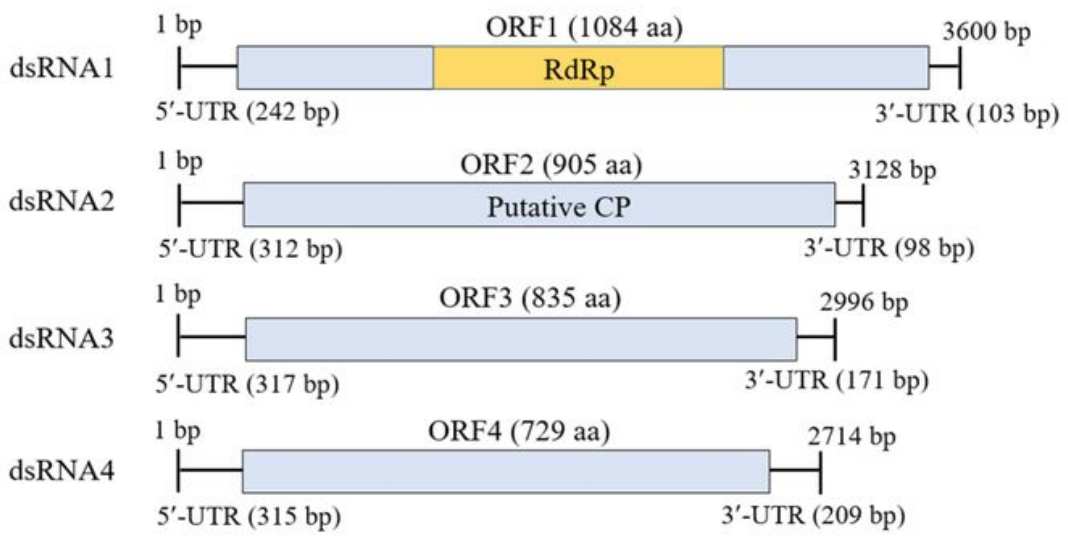

\section{C}

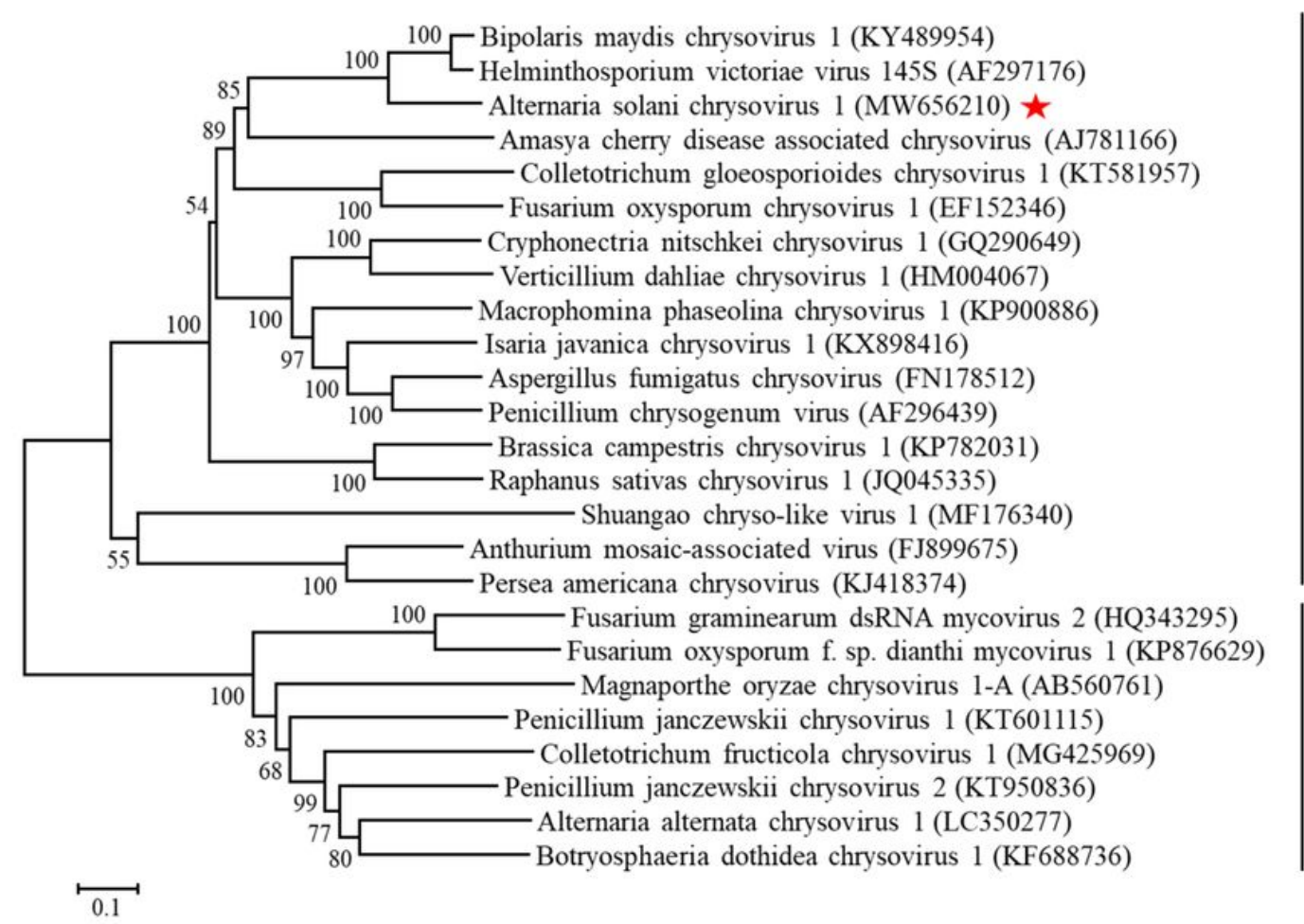

Alphachrysovirus

Betachrysovirus

\section{Figure 1}

Agarose gel electrophoresis, genomic organization, and phylogeny of Alternaria solani chrysovirus 1 (AsCV1) from phytopathogenic Alternaria solani strain DT-10. (A) The dsRNAs from A. solani strain DT-10 were resolved in 1.0\% agarose gel electrophoresis. Lane M, DL 5,000 DNA Marker; Lane 1, dsRNAs extracted from A. solani strain DT-10 and treated with S1 nuclease and DNase I. (B) Schematic diagram of the genome organization of AsCV1. The 5'-untranslated regions (UTRs), 3'-UTRs, and open reading frames (ORFs) were noted. The yellow region represents the conserved domain of RNA-dependent RNA polymerase (RdRp). (C) A phylogenetic tree based on the RdRp sequences of AsCV1 and 24 members of the family Chrysoviridae. The tree was constructed by neighbor-joining algorithm with bootstrapping analysis of 1000 replicates in MEGA6.0, and bootstrap values higher than $50 \%$ were shown. The scale bar represents a genetic distance of 0.1 , and the red star indicates AsCV1. 
dsRNA1 (Identity: $61.58 \%$ )

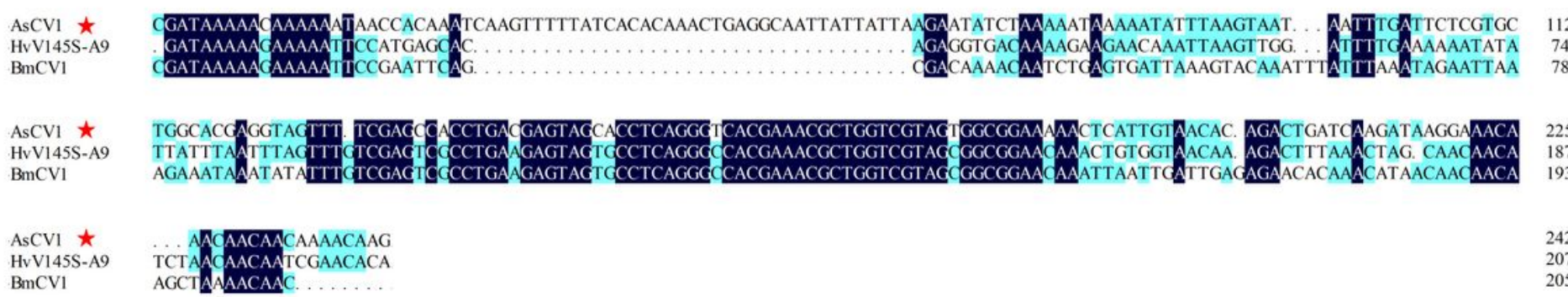

dsRNA2 (Identity: 68.01\%)

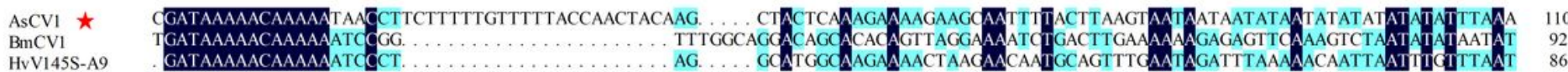

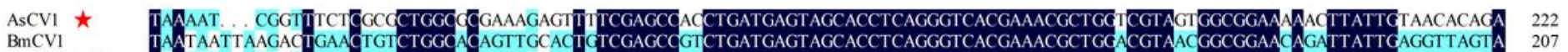

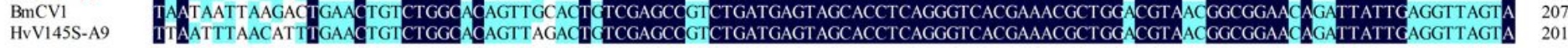

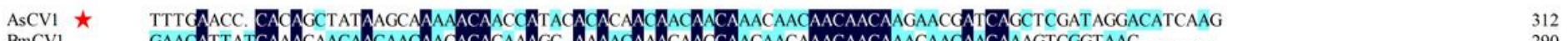

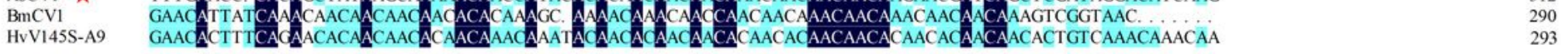

dsRNA3 (Identity: 66.97\%)

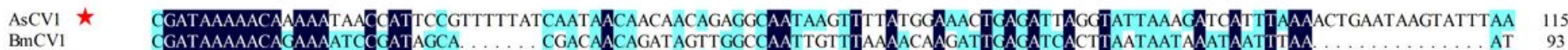

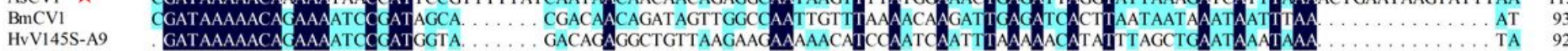

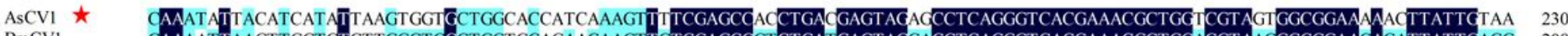

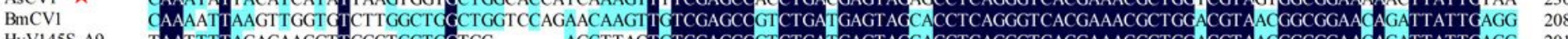

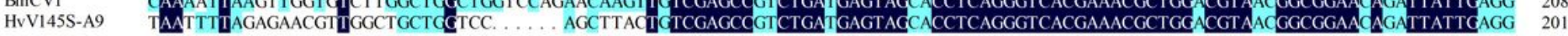

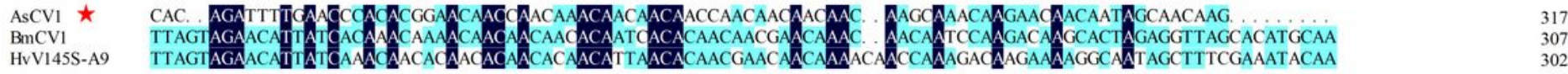

\section{dsRNA4 (Identity: 51.65\%)}

AsCVI $\star$

$\mathrm{BmCV1}$

HvV145S-A9

$\mathrm{AsCV} 1 \star$

BmCV1

HvV145S-A9

GATAA

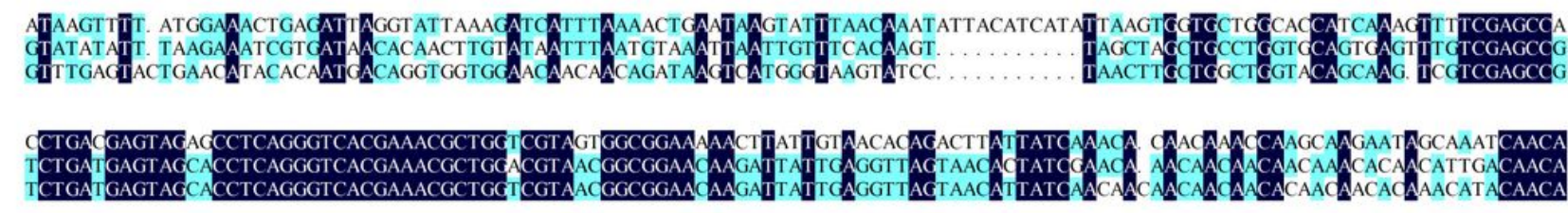

CGATAAAAACAAAATAACCATTCCGTTTTT ATIAATAACAACAACAGAGGCA TGATAAAAACACAAAATCCGAATCGACAAACT AAACACGATTCAGAACATAG 52

$\mathrm{AsCVI} \star$

$\mathrm{BmCV1}$

HvV145S-A9

AsCV1 $\star$

BmCVI

HvV145S-A9

CAGA. ACAAACAACAGGAAACAAACATACAACAGG ...

CAACAACA ACAAACACAAGCACAACA ACAATTTCGTAGTTTGTCAGTTCTAAATAGGATCAACAAACATACAAGCAAAA.

Figure 2

Multiple sequences alignment of the 5'-untranslated regions (UTRs) in dsRNA1-4 of AsCV1, Helminthosporium victoriae virus 145S (HvV145S-A9), and Bipolaris maydis chrysovirus 1 (BmCV1). The colors of dark blue and green represent $100 \%$ and $67 \%$ nucleotide identity in shade, respectively. The red stars indicate AsCV1.

\section{Supplementary Files}

This is a list of supplementary files associated with this preprint. Click to download. 
- SequencedataofAlternariasolanichrysovirus1.txt

- SupplementaryFig.1.tif

- SupplementaryTable1.docx

- SupplementaryTable2.docx 